\title{
Opioid utility function: methods and implications
}

\section{Cornelis Jan van Dam, Marijke Hyke Algera, Erik Olofsen, Leon Aarts, Terry Smith, Monique van Velzen, Elise Sarton, Marieke Niesters, Albert Dahan}

Department of Anesthesiology, Leiden University Medical Center, Leiden, The Netherlands

Contributions: (I) Conception and design: All authors; (II) Administrative support: None; (III) Provision of study materials or patients: All authors; (IV) Collection and assembly of data: All authors; (V) Data analysis and interpretation: None; (VI) Manuscript writing: All authors; (VII) Final approval of manuscript: All authors.

Correspondence to: Prof. Dr. Albert Dahan, MD, PhD. Department of Anesthesiology, Leiden University Medical Center (H5-022), POBox 9600, 2300 RC Leiden, The Netherlands. Email: a.dahan@lumc.nl.

\begin{abstract}
Opioids are complex drugs that produce profit (most importantly analgesia) as well as a myriad of adverse effects including gastrointestinal motility disturbances, abuse and addiction, sedation and potentially lethal respiratory depression (RD). Consequently, opioid treatment requires careful evaluation in terms of benefit on the one hand and harm on the other. Considering benefit and harm from an economic perspective, opioid treatment should lead to profit maximization with decision theory defining utility as (profit - loss). We here focus on the most devastating opioid adverse effect, $\mathrm{RD}$ and define opioid utility $\mathrm{U}=\mathrm{P}$ (benefit) - $\mathrm{P}$ (harm), where $\mathrm{P}$ (benefit) is the probability of opioid-induced analgesia and $\mathrm{P}(\mathrm{harm})$ the probability of opioid-induced RD. Other utility functions are also discussed including the utility $\mathrm{U}=\mathrm{P}$ (benefit AND NOT harm), the most wanted opioid effect, i.e., analgesia without RD, and utility surfaces, which depict the continuum of probabilities of presence or absence of analgesia in combination with the presence or absence of RD. Utility functions are constructed from pharmacokinetic and pharmacodynamic data sets, although pragmatic utility functions may be constructed when pharmacokinetic data are not available. We here discuss utilities of several opioids including the partial mu-opioid-receptor agonist buprenorphine, the full opioid receptor agonists fentanyl and alfentanil, and the bifunctional opioid cebranopadol, which acts at mu-opioid and nociception/orphanin FQ-receptors. We argue that utility functions give clinicians the opportunity to make an informed decision when opioid analgesics are needed for pain relief, in which opioids with a positive utility function are preferred over opioids with negative functions. Furthermore, utility functions of subpopulations will give an extra insight as a utility functions measured in one subgroup (e.g., patients with postoperative pain, good opioid responders) may not be mirrored in other patient subgroups (e.g., neuropathic pain patients, poor opioid responders).
\end{abstract}

Keywords: Pain; opioid; benefit; harm; analgesia; respiratory depression (RD); utility; utility function

Submitted Oct 05, 2019. Accepted for publication Oct 26, 2019.

doi: 10.21037/apm.2019.10.09

View this article at: http://dx.doi.org/10.21037/apm.2019.10.09

\section{Introduction}

The United States and other Western countries face a devastating opioid epidemic due to a sharp increase in opioid prescriptions over the last decade. For example, in The Netherlands, a country with 17 million inhabitants, the number of individuals that received at least one opioid prescription was 260,000 ( $1.5 \%$ of the total population) in $2008,814,000$ in $2013(4.8 \%)$ and further increased to over 1 million in 2017 (6\%) (1,2). In the Netherlands, the reason for this exponential rise is likely to be due to the increase in opioid prescriptions for chronic noncancer pain at the expense of the use of non-steroidal anti-inflammatory drug (NSAIDs). The indication for an opioid prescription for non-cancer pain varies considerably but back pain, rheumatoid arthritis and fibromyalgia are 
among the most common risk factors (1). While the initial prescription opioid epidemic was followed in the United States by a second and third wave of illicit opioid epidemics with heroin and fentanyl as the most abused drugs, in The Netherlands such a disastrous development has not been observed as yet. Still, a large number of intoxications and fatalities were also reported in The Netherlands (1), most certainly related to opioid abuse, misuse and concomitant use of centrally acting depressants such as sleep medication, alcohol, antidepressants or antipsychotics.

Although the opioid epidemic has led to various programs aimed at curtailing wide-spread opioid prescription behavior (3), we strongly consider that opioid analgesics should indisputably remain the cornerstone of management of moderate to severe pain in perioperative patients, trauma care and the palliative setting. And, under specific well controlled conditions, we do not recommend to exclude the use of opioids in chronic non-cancer pain, albeit never as first choice. Irrespective of the indication, we believe that opioid treatment requires careful evaluation in terms of their intended benefit for the patient and possible adverse effects. For example, opioid therapy in patients with an addiction history requires careful supervision or is best avoided and alternative treatments should be pursued. In the end, the overall balance between positive and negative effects should always be directed towards a positive outcome.

\section{Utility function}

Opioid analgesics have many side effects including perceived reward that render them at high risk for abuse and addiction, in addition to gastro-intestinal motility disturbances, dizziness, and a potential life-threatening side effect, respiratory depression (RD). All of these side effects are important and all may compromise the quality of life of individual patients, and some may even endanger their lives. It is important to realize that different opioids differ significantly in their pharmacology with respect to intended and side effects profiles (4). Surprisingly, we know little about the influence of these differences in pharmacokinetics and pharmacodynamics among opioids on the complex interaction between analgesia and the multitude of side effects that patients may experience. We therefore propose the development and use of utility or safety functions to determine the opioid's toxicity in light of the amount or intensity of analgesia it produces (5).
The utility function is an economic concept from decision theory, in which utility = profit - loss $(6,7)$. Decision theory is applied in many scientific fields including economy, biology and social sciences (6). Also, in medicine and pharmacology decision theory is used; we use the definition utility = benefit - harm, where benefit is the wanted drug effect (e.g., pain relief) and harm the adverse effect (e.g., sedation, RD, death). In analogy with the statement made by Sheiner and Melmon for decisions made in medicine in general (7), we argue that the choice of drug treatment in acute and chronic pain medicine is often based on intuition and/or trial-and-error rather than on a formal consideration of benefit and harm. The use of formal analyses may help us in many ways, such as in making wellconsidered decisions, in planning a strategy for decisionmaking or in gaining appropriate knowledge to enhance the condition of the patient (7). It is important to realize that both benefit and harm will markedly differ among opioids, but also among patients and treatment settings. Hence, the opioid's utility is important and may not only be used in drug development (to develop the opioid with the most benefit and least harm), but may also be used to determine the best treatment option among different patient populations (and possibly even individual patients) and additionally may be used to determine the dose that ensures the largest benefit with as little as possible harm.

Traditionally, the safety of drugs is expressed by calculation of their therapeutic index (8). This is the ratio of two doses, one that produces toxic effects in $50 \%$ of the population and another that produces therapeutic effects in $50 \%$ of the population $\left(\mathrm{TD}_{50} / \mathrm{ED}_{50}\right)$. In animals, the therapeutic index is the ratio of the dose that produces lethality in $50 \%$ of animals over the dose that produces therapeutic effect in $50 \%$ of animals $\left(\mathrm{LD}_{50} / \mathrm{ED}_{50}\right)$. Evidently, a higher therapeutic index is preferred over a lower one. Still, one problem with the therapeutic index is that analgesic effect and toxic effect are described by dose- or concentration-effect relationships that are often not parallel due to the fact that different end-points have different mechanistic and transduction pathways. As a consequence, the therapeutic index becomes dose-dependent; the therapeutic index must be defined by comparing doses at one specific level of response such as $50 \%$ effect, which is a response level at the point of maximal slope and therefore greatest precision (8). Advantages of the utility function are that it converts two concentration-effect curves into a single function, either in the time domain (after one specific dose 
has been administered to the patient) or in the steady-state biophase (effect-site) concentration domain. Moreover, these utility functions may be calculated even when full dose (or concentration)-response curves have not been obtained.

\section{History [1978-2005]}

The first report of the utility function in medicine was by Sheiner and Melmon in 1978 on the safety of hypertension therapy (7). They performed an extensive analysis of the problem of hypertension and antihypertensive therapy in terms of benefit and harm and concluded that a totally precise utility function in the economic sense was not deemed possible due to the lack of sufficient and appropriate data (7). Still, they considered the concept to be useful to allow the patient to make an informed decision of whether to exchange drug-induced symptoms for a currently asymptomatic disease. The concept was later applied by Cullberg and colleagues in 2005 to determine the outcome of antithrombin therapy (9). They studied the pharmacokinetics and pharmacodynamics of the thrombin inhibitor melagatran in patients with acute deep vein thrombosis and estimated the utility $=$ (probability of thrombus regression) - (the probability of bleeding related events) as function of the melagatran area-under-thecurve (AUC). They observed that the estimated utility was positive and relatively flat over the entire AUC range (utility was +0.6 at an AUC $<1$ h.mM and decreased to +0.45 at an AUC of 13.5 h.mM). They further stated that selection of a relatively low dose is expected to provide an adequate safety margin, even in patients with low renal function, without the need for therapeutic drug monitoring (9). This is an excellent example of the use of the utility function to determine the dose that ensures therapeutic benefit with as little as possible harm.

We have already highlighted that opioids should not be considered as a homogenous class of drugs, as different pharmacokinetic and pharmacodynamic properties, together with complex and varied different pharmacological mechanisms, will result in different therapeutic and sideeffect outcomes $(4,10)$. Utility functions, therefore, will vary for different opioids. This may be illustrated first primarily in animal models by consideration of two physicochemically different opioids, buprenorphine and fentanyl, and a more recent opioid, cebranopadol, which exhibits multiple opioid-receptor interactions. Secondly, consideration of the utility function of analgesia versus RD in humans, for example with fentanyl and alfentanil, have led us to further the application of utility functions with the development of utility surfaces and pragmatic utility functions.

\section{Buprenorphine and cebranopadol}

In Leiden, we first applied the concept of the utility function in a rodent model to compare the safety of the opioid analgesics, fentanyl and buprenorphine (11). The probability of analgesia, $\mathrm{P}(\mathrm{A})$, minus the probability of $\mathrm{RD}, \mathrm{P}(\mathrm{RD})$, was estimated using pharmacokinetic and pharmacodynamic data. Over the biophase (effect-site) concentration range of $1-12 \mathrm{ng} / \mathrm{mL}$ the fentanyl utility was negative (minimum value $=-0.4$ at a biophase concentration of $8 \mathrm{ng} / \mathrm{mL}$ : i.e., the probability of $\mathrm{RD}$ exceeds that of analgesia). In contrast, the function was positive for buprenorphine (peak effect $=+0.75$ at a biophase concentration of $5 \mathrm{ng} / \mathrm{mL}$ : i.e., the probability of analgesia exceeds that of RD). At concentrations $>12 \mathrm{ng} / \mathrm{mL}$ the utility equaled zero indicative that the probability of both end-points was equal. Note that in this analysis, the continuous variables, analgesia and respiration, were transformed into dichotomous data (yes/no) allowing the definition of clear cutoffs or thresholds for presence/ absence of analgesia and RD, although this process will cause some loss of precision and power (8). The main reason for the difference in utility function polarity between fentanyl and buprenorphine lies in their mechanisms of action. Fentanyl is a full, selective mu-opioid receptor agonist. Agonist activity at the mu-receptor by fentanyl and similar drugs results in analgesic, RD, tolerance, dependence and abuse (12). In contrast, buprenorphine is a partial agonist at the mu-opioid receptor, antagonist at kappa-opioid receptor, and agonist at the nociception/ orphanin FQ-receptor (NOP). In the clinical dose range this produces an apparent ceiling in respiratory effect, even at full mu-opioid-receptor occupancy. A ceiling in analgesia with buprenorphine has not been observed in experimental human studies $(13,14)$. This ceiling in RD may be related to the partial agonism at the mu-opioid receptor or due to respiratory stimulation from activation of the NOP receptor. Consequently, the utility function indicates a greater probability of analgesia than RD for buprenorphine. This suggests overt advantages of buprenorphine over fentanyl. However, for this particular opioid (buprenorphine) additional drug-related characteristics should be considered as well. Buprenorphine displays slow receptor kinetics that make reversal with opioid receptor antagonists such 

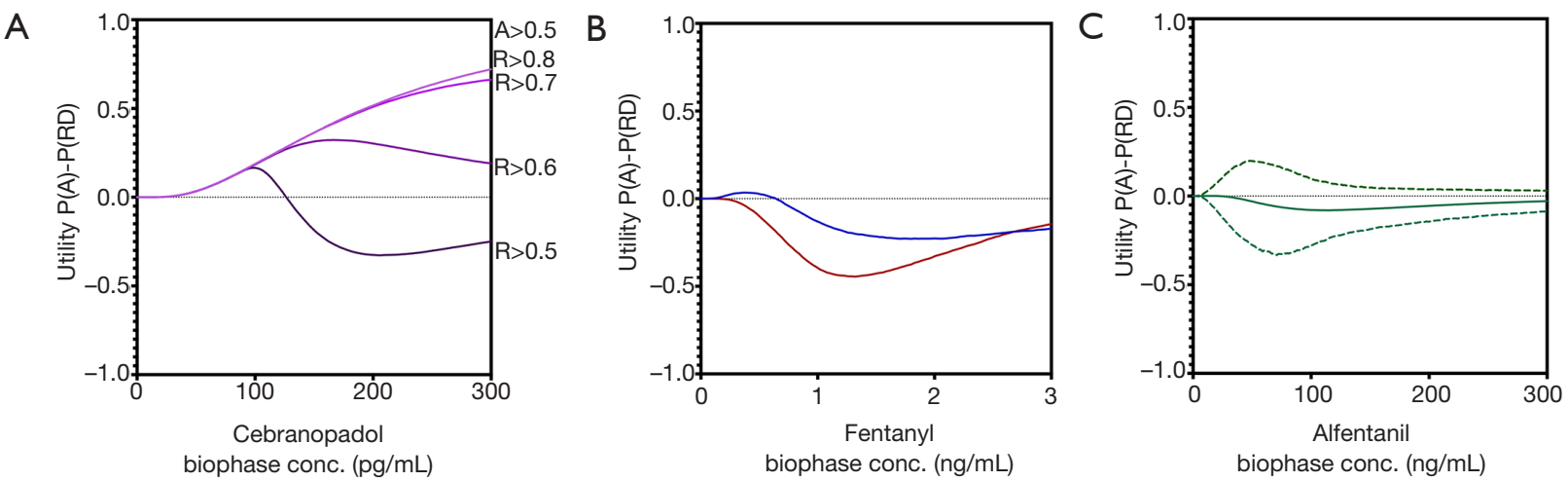

Figure 1 Utility function, $\mathrm{P}(\mathrm{A})$ - $\mathrm{P}(\mathrm{RD})$, as function of drug biophase concentration. (A) Cebranopadol utility function at one threshold for analgesia, $\mathrm{P}(\mathrm{A}>0.5)$ and different thresholds for respiratory depression: $\mathrm{P}(\mathrm{RD}>0.5), \mathrm{P}(\mathrm{RD}>0.6), \mathrm{P}(\mathrm{RD}>0.7)$ and $\mathrm{P}(\mathrm{RD}>0.8)$; $(\mathrm{B})$ Fentanyl utility function assessed by measuring respiratory depression and analgesia. Analgesia was assessed using transcutaneous electrical (blue line) and cutaneous heat pain (red line); (C) alfentanil utility function. Dotted lines are $\pm 95 \%$ confidence interval.

as with naloxone more difficult than for fentanyl with its rapid receptor kinetics $(11,15,16)$. Compared to fentanyl, about 10 -fold greater naloxone doses are required to reverse buprenorphine-induced $\mathrm{RD}$, best administered as continuous infusion to prevent renarcotization that will occur due to buprenorphine's long duration of action.

Cebranopadol is a novel synthetic opioid analgesic that activates all 4 opioid receptor subtypes, with high affinity for the NOP and mu-opioid receptors (Ki 0.7-0.8 nM) and lower affinities for the kappa- and delta-opioid receptors (Ki 3-18 nM) (17). Animal data indicate that simultaneous activation of the NOP and mu-opioid receptor may have the advantageous of increasing analgesic efficacy in a synergist fashion without increasing side effects, incl. RD (18). This may be due to an apparent ceiling in RD due to a respiratory stimulatory effect from NOP activation. The cebranopadol utility function is given in Figure 1A (17). Despite the overt positive animal data, the utility function becomes negative at biophase cebranopadol concentrations $>120 \mathrm{pg} / \mathrm{mL}$. This was a surprising observation but was corroborated by the $75 \%$ reduction of the hypercapnic ventilatory response slope following cebranopadol administration. Still, the drug is developed for treatment of neuropathic pain (and consequently may have its highest sensitivity in a neuropathic pain model) and lack of the applied electrical pain assay sensitivity may have influenced the utility function. Sensitivity analyses indicate that increasing cebranopadol potency (i.e., by testing the drug in a more sensitive nociceptive assay) or changing the threshold for analgesia (Figure 1A) steers the utility towards more positive values.

\section{Utility functions and utility surfaces of analgesia versus RD in humans}

\section{Fentanyl and alfentanil}

In 2013, we published the first systematic analysis of the fentanyl utility function derived from experiments in human volunteers (19). Following fentanyl pharmacokinetic and pharmacodynamic studies on analgesia (using an electrical pain threshold and tolerance model) and respiratory (using isohypercapnic ventilation) data, the utility function was calculated in time and concentration domains. The utility function $(\mathrm{U})$ as defined by us is context sensitive (Table 1):

$$
\begin{aligned}
& \mathrm{U}_{1}(\mathrm{C})=\mathrm{P}\left(\mathrm{A}>\mathrm{THR}_{\mathrm{A}}\right)-\mathrm{P}\left(\mathrm{RD}>\mathrm{THR}_{\mathrm{RD}}\right) \text {, and } \\
& \mathrm{U}_{2}(\mathrm{t})=\mathrm{P}\left(\mathrm{A}>\mathrm{THR}_{\mathrm{A}}\right)-\mathrm{P}\left(\mathrm{RD}>\mathrm{THR}_{\mathrm{RD}}\right)
\end{aligned}
$$

where $\mathrm{A}$ is analgesia and $\mathrm{RD}$ respiratory depression. The thresholds $\mathrm{THR}_{\mathrm{A}}$ and $\mathrm{THR}_{\mathrm{RD}}$ are the thresholds, or cutoff values, that separate presence of analgesia from absence of analgesia and presence of RD from absence of RD. In our main analysis we assumed that a $50 \%$ increase in stimulus intensity to reach pain threshold and tolerance) is an appropriate threshold for analgesia, while a decrease in isohypercapnic ventilation by $50 \%$ is appropriate for RD. To obtain the utility function 10,000 simulations for each of analgesia and RD were performed using the pharmacokinetic and pharmacodynamic parameter estimates. The occurrence of desired and toxic events was then counted and divided by 10,000 to obtain probabilities (Table 1). The utility function values range from -1 to +1 . The probability of greater analgesia than RD is defined by values of $U>0$, and vice versa. $U$ values $<-0.4$ and $>+0.4$ are considered large effects, while small effects (in between -0.2 
Table 1 Construction of the utility functions and surfaces based on pharmacokinetic-pharmacokinetic modeling studies

\begin{tabular}{|c|c|}
\hline Steps & Action \\
\hline Step 3 & $\begin{array}{l}\text { Perform 10,000 simulations for analgesia and toxic effects (in total 20,000 simulations) using parameter estimates and their } \\
\text { variability }\end{array}$ \\
\hline Step 4 & $\begin{array}{l}\text { Determine the probability of the occurrence of analgesia }(A) \text { and of respiratory depression }(R D) \text {, based on predefined } \\
\text { thresholds, such as: threshold }(A)=\text { at least } 50 \% \text { increase in pain tolerance, and threshold }(R D)=\text { at least a } 50 \% \text { reduction of } \\
\text { isohypercapnic minute ventilation }\end{array}$ \\
\hline Step 5 & $\begin{array}{l}\text { Define your utility and calculate the utility as function of time or as function of biophase concentration. Examples are: utility } \\
=\mathrm{P}(\mathrm{A}>50 \%)-\mathrm{P}(\mathrm{RD}>50 \%) \text {, cf. Figure } 1 \text {; utility }{ }_{2}=\mathrm{P}(\mathrm{A} \text { AND NOT RD })=\mathrm{P}(\mathrm{A}>50 \% \text { AND RD }<50 \%) \text {, cf. Figure } 2 A \text {; utility }{ }_{3}= \\
\mathrm{P}(\text { RD AND NOT } \mathrm{A})=\mathrm{P}(\mathrm{A}<50 \% \text { AND RD }>50 \%) \text {, cf. Figure } 2 B\end{array}$ \\
\hline
\end{tabular}

and +0.2 ) indicate absence of selectivity between the two end-points.

The fentanyl utility function in the concentration domain is shown in Figure $1 B$ for two distinct nociceptive endpoints, electrical pain tolerance (blue line) and thermal pain tolerance (red line). As is obvious from Figure 1B, the two assays differ in their sensitivity to fentanyl, with a greater sensitivity for the electrical pain model and consequently a more positive utility function. Irrespective of the model, the analyses indicate that the probability of RD exceeds the probability of analgesia over the studied biophase concentration effect range of 0 to $3 \mathrm{ng} / \mathrm{mL}$. We additionally performed a sensitivity analysis [data not shown, but see Figures 6 and 7 of Ref. (19)] to assess the effect of changes in context sensitivity (i.e., by applying different thresholds). The probability of greater analgesic effects than a $50 \%$ increase in pain tolerance (e.g., a $75 \%$ increase in pain tolerance) requires greater opioid efficacy, which is more difficult to attain at one specific opioid concentration [i.e., $\mathrm{P}(\mathrm{A})$ decreases] and additionally a concomitant greater probability of RD. Consequently, the utility function shifts to more negative values. A reduced threshold for RD (e.g., a $25 \%$ reduction in isohypercapnic ventilation) increases the probability of RD and consequently the value of the utility function becomes more negative. The importance of the choice of thresholds is evident from these examples. Further clinical studies are needed to determine the best set of thresholds that give the utility function an optimal clinical meaning.
Like fentanyl, alfentanil belongs to the family of phenylpiperidines. The alfentanil utility function, published in 2018, was not different from zero over the biophase concentration range of 0 to $300 \mathrm{ng} / \mathrm{mL}$ (Figure $1 C$; the dotted lines represent the $95 \%$ confidence interval of the utility function) (5). This suggests that the probability of $\mathrm{RD}$ for a given analgesic effect is slightly less for alfentanil compared to fentanyl. We further developed the concept of utility function by the construction of functions defined by the probability of analgesia without RD (i.e., the most desired condition in pain relief), $\mathrm{P}(\mathrm{A}$ AND NOT RD) (Figure $2 A$ ) and the probability of $\mathrm{RD}$ without analgesia (i.e., the least desired condition in pain relief), $\mathrm{P}(\mathrm{RD}$ AND NOT A) (Figure 2B). In a next step, we created so-called utility surfaces. Utility surfaces are a continuum of probabilities of presence or absence of analgesia in combination with the presence of absence of RD. The surfaces are plotted in $2 \mathrm{D}$, in which the various probability conditions are given a color ranking that range in between dark red (maximal probability of RD without analgesia) to bright green (maximal probability of analgesia without RD) (Figure $3 A$ ). This analysis allows a direct comparison between opioids considering the probabilities of two-end-points of interest. For example, comparing fentanyl and alfentanil confirms the somewhat greater respiratory effect of fentanyl over the clinical concentration range of the two opioids (fentanyl 0-3 ng/mL, alfentanil 0-300 ng/mL), with greater dark red combined with smaller bright green areas for fentanyl compared to alfentanil (Figure 3A,B). The reason for the 

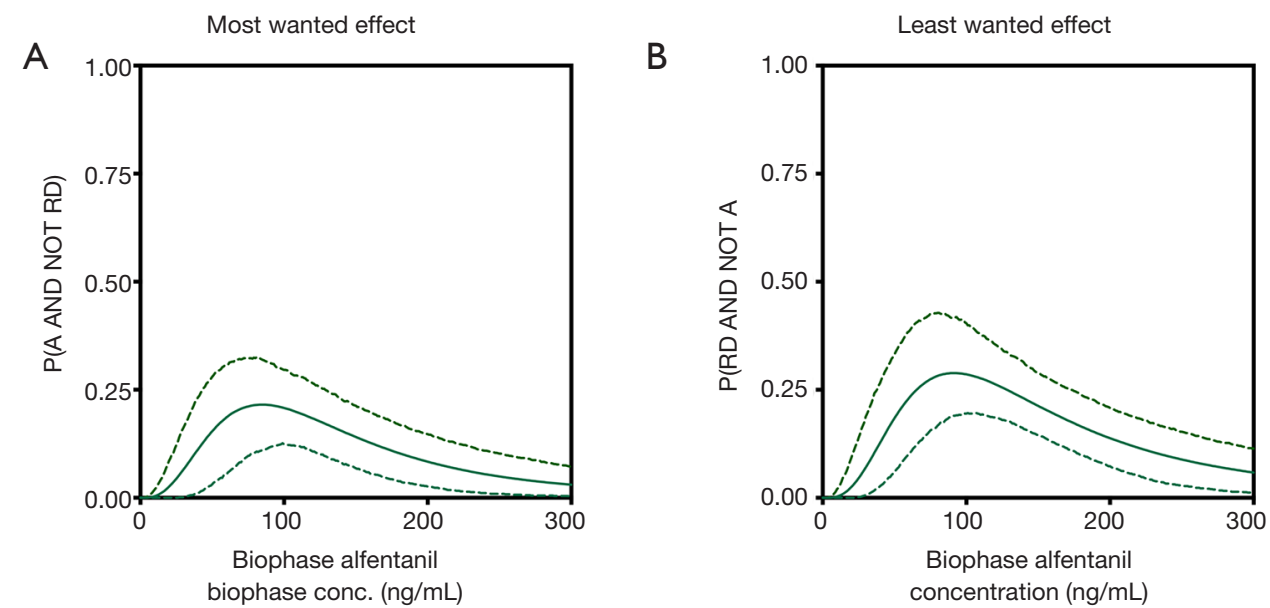

Figure 2 Probabilities of benefit without harm, P(A AND NOT RD) (A), and harm with benefit, P(RD AND NOT A) (B), of alfentanil as function of biophase drug concentration. Dotted lines are $\pm 95 \%$ confidence intervals.

A

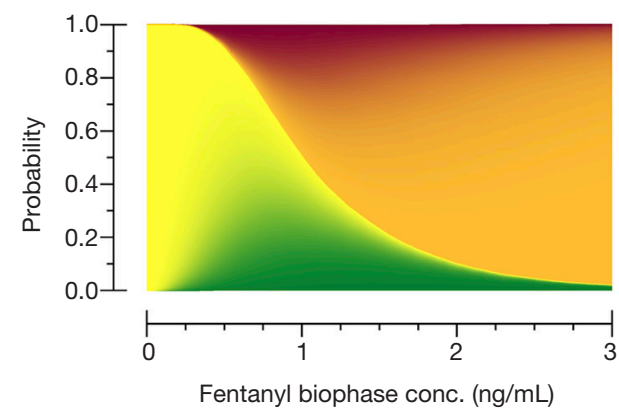

B

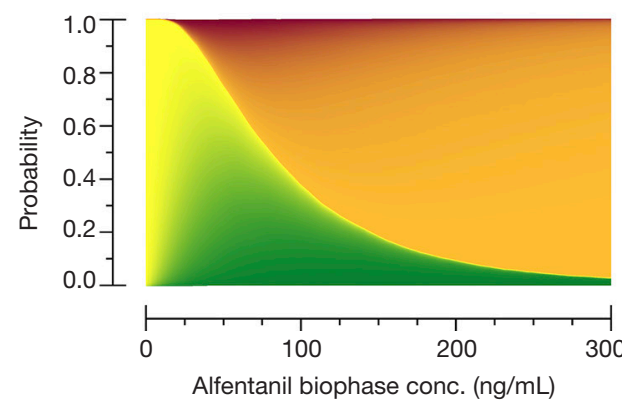

Figure 3 Utility surfaces of fentanyl (A) and alfentanil (B) as function of biophase concentration. The dark red surface indicates the area of high probability of respiratory without analgesia, the green surface the area of high probability of analgesia without respiratory depression. The fade towards yellow represents the reduction of the probability of analgesia towards zero (yellow area). The fade towards orange indicates the reduction of the probability of respiratory depression towards zero (orange area).

small differences in the respiratory behavior of these two tested phenylpiperidines remains unknown but is likely related to subtle differences in physicochemistry and/or pharmacokinetics and unrelated to possible differences in mechanistic pathways, although we cannot exclude that the two opioids differ in their ability to activate the two most important intracellular transduction pathways following mu-opioid receptor activation: the G-protein-related and the beta-arrestin-related pathways. Activation of the G-protein pathway is associated with analgesia, reward, and $\mathrm{RD}$, activation of the beta-arrestin pathway with nausea/ vomiting and RD (20,21). Recent studies indicate that bias towards the G-protein pathway will cause less respiratory effects (22) and enhanced antinociceptive effects (23).
Whether the opioids differ in this respect is not known. Still, the differences between the opioids were small and may have limited clinical relevance.

\section{The pragmatic utility function}

When drug concentrations are not measured, no pharmacokinetic model is available for the construction of the utility function. Since this occurs frequently, we developed 'pragmatic' utility functions that are based on measurements of benefit and harm but make no assumptions about the underlying pharmacokinetics and pharmacodynamics (24). To that end, both harm (RD) and benefit (A) are treated as binary outcomes (they occur: 
yes $=1$ or no $=0$ ). The probability $\mathrm{p}$ that one or the other conditions occurs is estimated by the proportion denoted by $\mathrm{P}$ in a population of $\mathrm{n}$ subjects. So, $\mathrm{P}(\mathrm{A})$ is calculated as $\mathrm{n}(\mathrm{A}$ $=1) / n($ total $)$ and $P(R)=n(R D=1) / n($ total $)$; the variance of the proportion is given by $\operatorname{var}(\mathrm{P})=\mathrm{P}(1-\mathrm{P}) / \mathrm{n}$. We tested this pragmatic function for the toxic effects (RD or sedation) of fentanyl and the non-opioid analgesic pregabalin. For both drugs robust and useful (i.e., pragmatic) utility functions and surfaces were obtained that closely mimic the utility curves derived from the more elaborate analysis given in Table 1. Observed differences are related to the lack of residual error, causing increased uncertainty in the pragmatic approach.

\section{Conclusions and future directions}

Opioids remain the cornerstone of modern-day treatment of moderate to severe acute, perioperative and chronic cancer pain. Of all pain relief therapies, opioids are most efficacious, especially on the short run. Opioids produce severe adverse effects that may produce harm to the patient and consequently limit patient and doctor compliance. Some of these effects are acceptable, especially when they are relatively minor compared to the wanted effects, others are devastating and/or potentially life-threatening (addiction, sedation, RD, cardiorespiratory collapse). Importantly, the spectrum of benefit and harm differs, not only among different opioids, but also among different patient populations and pain syndromes. For example, opioid efficacy is high in postoperative pain but is limited in neuropathic pain, and is lost in opioid-tolerant patients. It is often not straightforward how to concomitantly consider benefit and harm of treatment considering the fact that different concentration- or dose-effect relationships may exist for the different opioid end-points (benefit versus toxic effects) and adverse effects may vary over time with some increasing and others vanishing during the treatment period.

In order to capture opioid benefit and harm into a single function, we developed utility functions and utility surfaces. These functions give an indication of the probability of benefit (analgesia) and the probability of harm (e.g., sedation or RD) and their combination [P(A AND RD), $\mathrm{P}(\mathrm{A}$ AND NOT RD), etc.]. The benefit of this approach is that it is now relatively easy to compare opioids in terms of their adverse effects for a given amount of analgesic effect. Additionally, within specific populations, opioid behavior may be studied in detail and lead to a better mechanistic understanding of differences between patient subpopulations. For example, all perioperative clinicians are aware of the fact that some patients require excessive amounts of opioids before adequate analgesic levels are reached. These, so-called poor responders contrast patients that require just low doses of an opioid until adequate analgesia. Aubrun et al. calculated a 40 -fold difference in postoperative opioid requirements among patients (25). No information exists on the balance between analgesia and toxic opioid effects in these poor and good opioid responders. Utility functions of these two populations are insightful in this respect (Figure 4) (26). Good responders exhibit a positive utility function indicative that analgesic efficacy does not translate into a high probability of RD. However, poor responders do have a negative utility function, indicative that the low probability of analgesia coincides with a high probability of RD. These differences among subpopulations may possibly be related to the differences in activation of distinct transduction pathways for analgesia and RD (G-proteins and beta-arrestin, see above). Current studies of utility functions have focused on comparison between the analgesic (benefit) and respiratory depressant (harm) properties of the opioids. However, similar approaches may be taken toward the comparison of analgesia (benefit) and addictive properties (harm), which could have a significant bearing on the therapeutic use of opioids in chronic pain, potentially avoiding a concomitant rise in drug dependency and illicit use. Opioid tolerance and dependence appear to be associated with opioid receptor desensitization, an opioid effect again reliant on G-protein and beta-arrestin mechanistic pathways (27).

Finally, the importance of utility functions further lies in the development of new opioids in for example phase 2 and 3 trials (8). Opioids with positive utility functions are evidently preferred over opioids with negative functions. Additionally, diverse patient populations need to be tested as a positive function for one indication (e.g., postoperative pain) may not be mirrored in another patient subgroup (e.g., patients with sleep-disordered breathing, neuropathic pain patients, patients with mu-opioid receptor mutations).

Future studies are aimed at the validation of the utility function in clinical practice, for example in postoperative pain patients. To that end, we will determine the utility value, $\mathrm{P}(\mathrm{A})$ - $\mathrm{P}(\mathrm{RD})$, at the occurrence of an individual respiratory event. We expect that most events will occur at relatively low utility values, such as below +0.2 , when the probability of harm is relatively high. Still, we do not exclude the occurrence of events at moderately high utility 


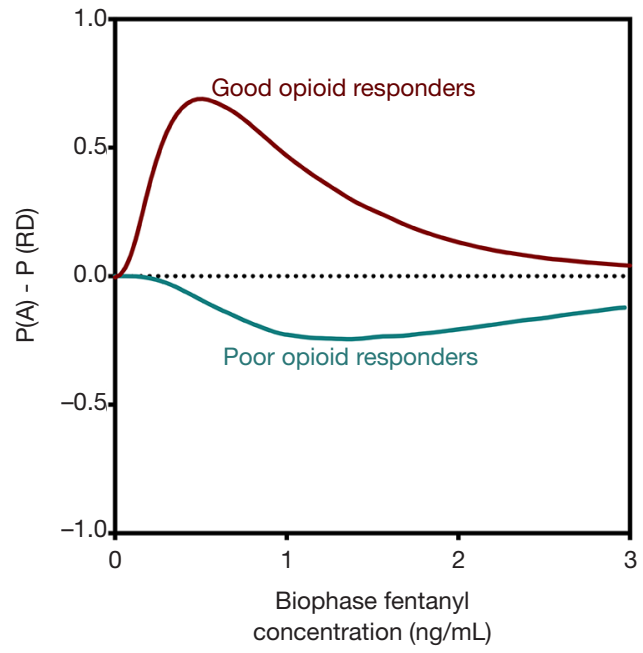

Figure 4 Utility function, $\mathrm{P}(\mathrm{A})$ - P (RD), as function of biophase fentanyl concentration in good responders (subjects with high efficacy from already low doses of fentanyl), and poor responders (subjects that require high fentanyl doses before analgesia sets in).

values, for example from +0.2 to +0.4 . Pain in real life fluctuates and when both probabilities of benefit and harm are high and the $\mathrm{P}(\mathrm{A})-\mathrm{P}(\mathrm{R})$ utility value is between +0.2 and +0.4 , and pain is absent, the moderate utility value may still be insufficiently protective. These studies are currently underway.

\section{Acknowledgments}

Funding: None.

\section{Footnote}

Provenance and Peer Review: This article was commissioned by the Guest Editor (Mellar P. Davis) for the series "Opioid Utility the Other Half of Equianalgesia" published in Annals of Palliative Medicine. The article was sent for external peer review organized by the Guest Editor and the editorial office.

Conflicts of Interest: The series "Opioid Utility the Other Half of Equianalgesia" was commissioned by the editorial office without any funding or sponsorship. The authors have no other conflicts of interest to declare.

Ethical Statement: The authors are accountable for all aspects of the work in ensuring that questions related to the accuracy or integrity of any part of the work are appropriately investigated and resolved.

Open Access Statement: This is an Open Access article distributed in accordance with the Creative Commons Attribution-NonCommercial-NoDerivs 4.0 International License (CC BY-NC-ND 4.0), which permits the noncommercial replication and distribution of the article with the strict proviso that no changes or edits are made and the original work is properly cited (including links to both the formal publication through the relevant DOI and the license). See: https://creativecommons.org/licenses/by-nc-nd/4.0/.

\section{References}

1. Bedene A, Lijfering WM, Niesters M, et al. Opioid Prescription Patterns and Risk Factors Associated with Opioid Use in the Netherlands. JAMA Netw Open 2019;2:e1910223.

2. Dahan A, Kramers K, Schellekens A, et al. Pain control: give opioids only a supporting role [in Dutch]. Available online: https://www.medischcontact.nl/nieuws/laatstenieuws/artikel/pijnbestrijding-geef-opiaten-slechtseenbijrol.htm. Accessed March 22, 2019.

3. Pardo B. Do more robust prescription drug monitoring programs reduce prescription opioid overdose? Addiction 2017;112:1773-83.

4. Drewes AM, Jensen RD, Droney J, et al. Differences between opioids: Pharmacological, experimental, clinical and economical perspectives. Br J Clin Pharmacol 2013;75:60-78.

5. Roozekrans M, van der Schrier R, Aarts L, et al. Benefit versus severe side effects of opioid analgesia: novel utility functions of probability of analgesia and respiratory depression. Anesthesiology 2018;128:932-42.

6. Habibi I, Cheong R, Lipniacki T, et al. Computation and measurement of cell decision making errors using single cell data. PLoS Comput Biol 2017;13:e1005436.

7. Sheiner LB, Melmon KL. The utility function of antihypertensive therapy. Ann N Y Acad Sci 1978;304:112-27.

8. Kharasch ED, Rosow CE. Assessing the utility of the utility function. Anesthesiology 2013;119:504-6.

9. Cullberg M, Eriksson UG, Wåhlander K, et al. Pharmacokinetics of ximelagatran and relationship to clinical response in acute deep vein thrombosis. Clin Pharmacol Ther 2005;77:279-90.

10. Badal S, Turfus, S, Rajnarayanan R, et al. Analysis 
of natural product regulation of opioid receptors in the treatment of human disease. Pharmacol Ther 2018;184:51-80.

11. Yassen A, Olofsen E, Dahan A, et al. Pharmacokineticpharmacodynamic modeling of the efficacy and safety of buprenorphine in rats. Pharm Res 2008;25:183-93.

12. Armenian P, Vo KT, Barr-Walker J, et al. Fentanyl, fentanyl analogues and novel synthetic opioids: a comprehensive review. Neuropharmacology 2018;134:121-32.

13. Yassen A, Olofsen E, Romberg R, et al. Mechanism based $\mathrm{PK} / \mathrm{PD}$ modeling of the respiratory depressant effect of buprenorphine and fentanyl in healthy volunteers. Clin Pharmacol Ther 2007;81:50-8.

14. Dahan A, Yassen A, Bijl H, et al. A comparison of the respiratory effects of intravenous buprenorphine and fentanyl in humans and rats. Br J Anaesth 2005;94:825-34.

15. Yassen A, Olofsen E, Bijl H, et al. Mechanism-based pharmacokinetic/pharmacodynamic modeling of the antinociceptive effect of buprenorphine in healthy volunteers. Anesthesiology 2006;104:1232-42.

16. Algera MH, Kamp J, van der Schrier R, et al. Opioid-induced respiratory depression in humans: pharmacokinetic-pharmacodynamic modelling of reversal. Br J Anaesth 2019;122:e168-79.

17. Dahan A, Boom M, Sarton E, et al. Respiratory effects of the nociceptin/orphanin FQ peptide and opioid receptor agonist, cebranopadol, in healthy human volunteers. Anesthesiology 2017;126:697-707.

18. Linz K, Schröder W, Frosch S, et al. Opioid-type respiratory depressant side effects of cebranopadol in rats are limited by Its nociceptin/orphanin FQ peptide receptor agonist activity. Anesthesiology 2017;126:708-15.

Cite this article as: van Dam CJ, Algera MH, Olofsen E, Aarts L, Smith T, van Velzen M, Sarton E, Niesters M, Dahan A. Opioid utility function: methods and implications. Ann Palliat Med 2020;9(2):528-536. doi: 10.21037/apm.2019.10.09
19. Boom M, Olofsen E, Neukirchen M, et al. Fentanyl Utility Function: A risk-benefit composite of pain relief and breathing responses. Anesthesiology 2013;119:663-74.

20. Violin JD, Lefkowitz RJ. b-Arrestin-biased ligands at seven-transmembrane receptors. Trends Pharmacol Sci 2007;28:416-22.

21. Siuda ER, Carr R, Rominger DH, et al. Biased mu-opioid receptor ligands: a promising new generation of pain therapeutics. Curr Opin Pharmacol 2017;32:77-84.

22. Soergel DG, Subach RA, Burnham N, et al. Biased agonism of the 1-opioid receptor by TRV130 increases analgesia and reduces on-target adverse effects versus morphine: A randomized, double-blind, placebocontrolled, crossover study in healthy volunteers. Pain 2014;155:1829-35.

23. Mori T, Kuzumaki N, Arima T, et al. Usefulness for the combination of $G$ protein- and $\beta$-arrestin-biased ligands of $\mu$-opioid receptors: Prevention of antinociceptive tolerance. Mol Pain 2017;13:1744806917740030.

24. Olesen AE, Broens S, Olesen SS, et al. A Pragmatic Utility Function to Describe the Risk-Benefit Composite of Opioid and Nonopioid Analgesic Medication. J Pharmacol Exp Ther 2019;371:416-21.

25. Aubrun F, Salvi N, Coriat P, et al. Sex- and age-related differences in morphine requirements for postoperative pain relief. Anesthesiology 2005;103:156-60.

26. Dahan A, Olofsen E, Niesters M. Pharmacotherapy for Pain: Efficacy and Safety Issues Examined by Subgroup Analyses. Pain 2015;156 Suppl 1:S119-26.

27. Listos J, Lupina M, Talarek S, et al. The mechanisms involved in morphine addiction: an overview. Int J Mol Sci 2019. doi: 10.3390/ijms20174302. 